\title{
A Points Imaging Passive Localization Algorithm for Bistatic Stations in the Parallel Track and Squint Mode
}

\author{
Longjian Zhou, a , Jingqing Luo, b , Shilong $\mathrm{Wu}^{1}$ \\ ${ }^{1}$ Electronic Engineering Institute, Hefei, 230037, China \\ aemail: zhoulj007@126.com, bemail:Luojingqing001@126.com
}

\begin{abstract}
Keywords: Passive Localization; Time Difference of Arrival; Frequency Difference of Arrival; Direct Position Determination; Synthetic Aperature Radar
\end{abstract}

\begin{abstract}
For passive localization of wideband signal sources, a points imaging passive localization algorithm is proposed with the information of time difference of arrival(TDOA) and frequency difference of arrival(FDOA). This method uses the principle of Synthetic Aperature Radar(SAR) imaging and the idea of direct position determination(DPD) for reference. The observing time is divided into fast time and slow time in sampling. On the basic of analyzing range difference of arrival(RDOA) walking in the parallel track and squint mode, the RDOA walking is corrected in fast time, and the FDOA spatial variation is compressed. Then joint TDOA and FDOA are estimated and mapped to the position of target. The results of simulation prove the effectiveness of the proposed algorithm. The proposed algorithm is especially applicable for the observing unresolved multiple targets localization with properties of high precision and super resolution.
\end{abstract}

\section{Introduction}

Passive localization has become a hot area of research with the merits as electromagnetic self-hiding, far distance detection. With the rapid development of electromagnetic countermeasure (ECM), active decoy radar is usually placed around key target emitters to achieve anti-reconnaissance and anti-interference. The active decoy radar site is consistent with key target emitter location. In addition, both time duration and bandwidth of active decoy radar are often slightly above these of key target radar signal. When key target radar signal buried in decoy radar signal that the presence of the observation of multiple unresolved targets, the traditional localization method become impotent. The observation of multiple unresolved targets refers to sample signal and observational parameter cannot correspond to target during the observation period, for example, when acquiring mixed signals of multiple emitters on a time slice, these signals may be in the same reconnaissance beam as well as overlapping in time and frequency domain and the sampled signal cannot be determined from which one target or which several targets.

The traditional passive location method ${ }^{[1-3]}$ is to observe the target of direction of arrival (DOA), the time difference of arrival (TDOA) and the frequency difference of arrival (FDOA) multiple times for localization, however, there must be problems of signal sorting and parameter pairing during the process of localization. In recent years, Anthony J. Weiss have proposed direct position determination (DPD), which uses sampling signals directly instead of parameter measurements to overcome the shortcomings of signal sorting and parameter pairing in the traditional "two-step" localization method, and also possesses better positioning accuracy than traditional method in low signal-to-noise ratio (SNR) images. The maximum likelihood estimation algorithm of single narrowband target emitter in the case of multiple observatories is proposed by references 4 . References 5 introduced the advantages of direct position determination, which was also analyzed in the case of multi-path effect, array error and model error by mutual coupling in the references 6 . In addition, the direct position determination under multiple emitters was studied in references 7 . References 8 and 9 dealt with the direct position determination of maximum likelihood estimation based on TDOA and FDOA. References 8 and 9 used deterministic unknown signal and wide-sense stationary Gaussian stochastic signal as signal models, respectively, however, both two signal 
models haven not considered the impact of range walk to arrival times, moreover, the compensation of TDOA and FDOA had high calculation and low time efficiency. In references 10 and 11, maximum likelihood estimation algorithm was studied, the transformation relationship between Cross Ambiguity Matrix (CAM) and Cross Ambiguity Function (CAF) was established, which resulted in simplified calculation, but it still requires multidimensional search. The direct position determination based on sparse representation was researched in references 13 to 15, of which direct location algorithm of known signal and mapping observed signal into observation region to avoid the problem of signal transfers by references 13. References 14 optimized the multi-goal location by approximate $l_{0}$ norm. According to localization problems of deterministic unknown signal, references 15 estimated signal by Minimum Variance Unbiased estimator (MVU), then represented estimation for target location by sparse construction, but there was still large computational capacity for passive localization based on unknown signal.

From a localization perspective, imaging is another form of localization. SAR used the correlation of echo signals to improve the vertical upward resolution of beam through cumulating Doppler frequency caused by relative motions between emitter and reconnaissance receiver, and to achieve equivalent radar aperture synthesizing in the direction of motion and azimuth high-resolution imaging[16-18]. SAR imaging algorithms mainly contain Range-Doppler (R-D) algorithm, Omega-k algorithm ( $\omega-\kappa)$, Chirp Scaling (CS) algorithm and Back-Projection (BP) algorithm. In recent years, many scholars have studied Bistatic Synthetic Aperture Radar(Bi-SAR) from various perspectives ${ }^{[19]}$. Bi-SAR installed transmitters on the platform with relatively high flight altitude and then installed transmitters on the platform at low altitude with relatively high maneuverability, and achieved to image observation region. Similarly, by utilizing the sampled data, SAR estimated the arrival times in the fast time domain and corrected range walk, as well as estimating the Doppler frequency in the slow time domain and mapping to two dimensional planes for imaging. If time of arrival and Doppler frequency are considered as location coordinates, SAR will be a special direct position.

To avoid multidimensional search with large computational complexity in the calculation process of direct position determination, the observing time is divided into fast time and slow time in sampling on the basis of analyzing range difference of arrival (RDOA) walking in the parallel track and squint mode by using SAR imaging principle. Based on sampling pulse data, the RDOA walking was corrected as well as estimating the TDOA in fast time and the TDOA of pulse signals in observing time were obtained. In the slow time, the FDOA spatial variation was compressed. Then joint TDOA and FDOA were estimated and mapped to the position of targets. Compared with Bi-SAR, which is positioned by the sum of range and the sum of Doppler frequency of two reconnaissance devices arrival, the proposed approach uses two reconnaissance devices of RDOA and FDOA for localization. Comparing this method to the traditional 'two-step' positioning, it fuses information of pulse signals in observing time, then estimates TDOA and FDOA jointly and mapped to the position of target that the obtained TDOA and FDOA could be considered as another representation of target location. In addition, the observing time is divided into fast time and slow time in sampling and the RDOA walking is corrected in fast time and the FDOA spatial variation is compressed in slow time, then joint TDOA and FDOA are estimated in this method compared with classical CAF, which carries out the joint estimation to TDOA and FDOA in the total observation time (or pulse time) without RDOA walk correction and FDOA spatial variation.

\section{Signal Model}

Figure 1 presents the operation diagram of parallel track and squint mode. Reconnaissance device $P_{1}$, which based on $\boldsymbol{X}_{P_{1}}(0)=\left[-L, 0, H_{1}\right]^{\mathrm{T}}$, travels at the speed of $v_{1}$ on the $\mathrm{Y}$ axis. Reconnaissance device $P_{2}$ lies at $\boldsymbol{X}_{P_{2}}(0)=\left[L, 0, H_{2}\right]^{\mathrm{T}}$ and flies at the speed of $v_{2}$ on the Y axis. $E_{o}$, whose coordinate is $\boldsymbol{X}_{o}=\left[x_{o}, y_{o}, 0\right]^{\mathrm{T}}$, is the central of observation area. $E_{T}$ is the target location 
and its coordinate is $\boldsymbol{X}_{T}=\left[x_{T}, y_{T}, 0\right]^{\mathrm{T}}$.

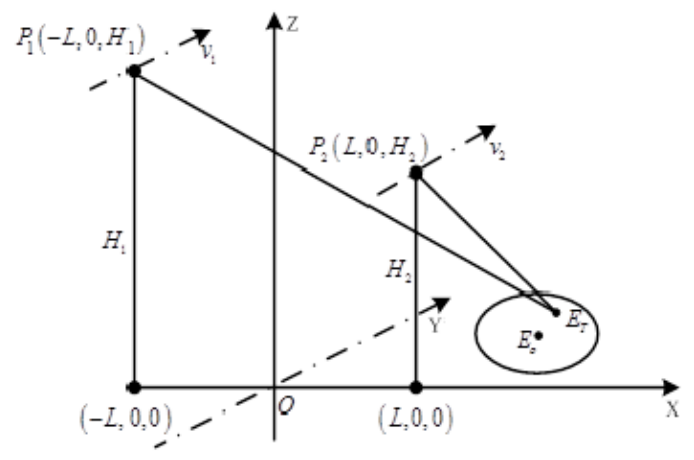

Fig.1 The operation diagram of parallel track and squint mode

At the moment of $t_{m}$, reconnaissance devices $P_{1}$ and $P_{2}$ locate at $\boldsymbol{X}_{P_{1}}\left(t_{m}\right)=\left[-L, v_{1} t_{m}, H_{1}\right]^{\mathrm{T}}$ and $\boldsymbol{X}_{P_{2}}\left(t_{m}\right)=\left[L, v_{2} t_{m}, H_{2}\right]^{\mathrm{T}}$, respectively. Then at this time of $t_{m}$, the distances of emitter to these two reconnaissance devices is:

$$
\begin{aligned}
R_{1}\left(t_{m}\right) & =\sqrt{r_{1}^{2}+\left(v_{1} t_{m}-y_{T}\right)^{2}} \\
R_{2}\left(t_{m}\right) & =\sqrt{r_{2}^{2}+\left(v_{2} t_{m}-y_{T}\right)^{2}}
\end{aligned}
$$

respectively,

where

$$
\begin{aligned}
& r_{1}^{2}=\left(-L-x_{T}\right)^{2}+H_{1}^{2} \\
& r_{2}^{2}=\left(L-x_{T}\right)^{2}+H_{2}{ }^{2} .
\end{aligned}
$$

Assume that the envelope of the $m$-th pulse signal by target emitter is $s_{m}\left(t_{r}, t_{m}\right)$ and the carrier frequency of this signal is $f_{c}$ and then down-convert this signal to $f_{i}$, the received $m$-th pulse signals of reconnaissance devices $P_{1}$ and $P_{2}$ can be written as:

$$
\begin{aligned}
& x_{1}\left(t_{r}, t_{m}\right)=s_{m}\left(t_{r}-\tau_{1}\left(t_{m}\right), t_{m}\right) e^{j 2 \pi f_{i}\left(t_{m}+t_{r}\right)} e^{-j 2 \pi f_{c} \tau_{1}\left(t_{m}\right)} \\
& x_{2}\left(t_{r}, t_{m}\right)=s_{m}\left(t_{r}-\tau_{2}\left(t_{m}\right), t_{m}\right) e^{j 2 \pi f_{i}\left(t_{m}+t_{r}\right)} e^{-j 2 \pi f_{c} \tau_{2}\left(t_{m}\right)}
\end{aligned}
$$

respectively,

where

$$
\begin{aligned}
\tau_{1}\left(t_{m}\right) & =\frac{R_{1}\left(t_{m}\right)}{c} \\
\tau_{2}\left(t_{m}\right) & =\frac{R_{2}\left(t_{m}\right)}{c} .
\end{aligned}
$$

\section{Analysis of RDOA Walking}

Let the RDOA of receiving signals from two reconnaissance devices at $t_{m}$ be expressed as:

$$
R\left(t_{m}\right)=R_{1}\left(t_{m}\right)-R_{2}\left(t_{m}\right)
$$

Let $R_{10}=R_{1}(0), \quad R_{20}=R_{2}(0)$. In the case of $v_{1} t_{m} \ll R_{10}$ and $v_{2} t_{m} \ll R_{20}$, by second order Tailor expansion, formulas (1) and (2) can be approximated as:

$$
\begin{aligned}
& R_{1}\left(t_{m}\right) \approx R_{10}-\frac{y_{T} v_{1}}{R_{10}} t_{m}+\frac{r_{1}^{2} v_{1}{ }^{2}}{2 R_{10}{ }^{3}} t_{m}{ }^{2} \\
& R_{2}\left(t_{m}\right) \approx R_{20}-\frac{y_{T} v_{2}}{R_{20}} t_{m}+\frac{r_{2}{ }^{2} v_{2}{ }^{2}}{2 R_{20}{ }^{3}} t_{m}{ }^{2}
\end{aligned}
$$


respectively.

Next formulas (6) and (7) are substituted to formula (5), we have:

$$
R\left(t_{m}\right) \approx R_{0}+\Delta R\left(t_{m}\right)
$$

where

$$
\begin{gathered}
R_{0}=R_{10}-R_{20} \\
\Delta R\left(t_{m}\right)=\eta t_{m}+\gamma t_{m}{ }^{2}
\end{gathered}
$$

and

$$
\begin{gathered}
\eta=-\frac{y_{T} v_{1}}{R_{10}}+\frac{y_{T} v_{2}}{R_{20}} \\
\gamma=\left(\frac{r_{1}^{2} v_{1}^{2}}{2 R_{10}{ }^{3}}-\frac{r_{2}^{2} v_{2}^{2}}{2 R_{20}{ }^{3}}\right) .
\end{gathered}
$$

From the formula (10) can be seen, there are spatial variance of RDOA walking when TDOA and FDOA are joint estimation positioned. The RDOA walking momentum is $\Delta R\left(t_{m}\right)$, the RDOA walking rate is $\eta$, the FDOA walking momentum is approximately $2 \gamma t_{m}$ and the FDOA walking rate is $\gamma$. Figure 2 and 3 give the variation of the RDOA and FDOA at some typical parameters (accords with single target in simulation analysis) with slow time $t_{m}$. If pulse signals are sampled at $100 \mathrm{MHz}$, the RDOA walking can span 23 range cells and the FDOA varies by about $218 \mathrm{~Hz}$, in addition, defocus will occur when TDOA and FDOA are estimated jointly, so the RDOA walking and the FDOA walking must be corrected.

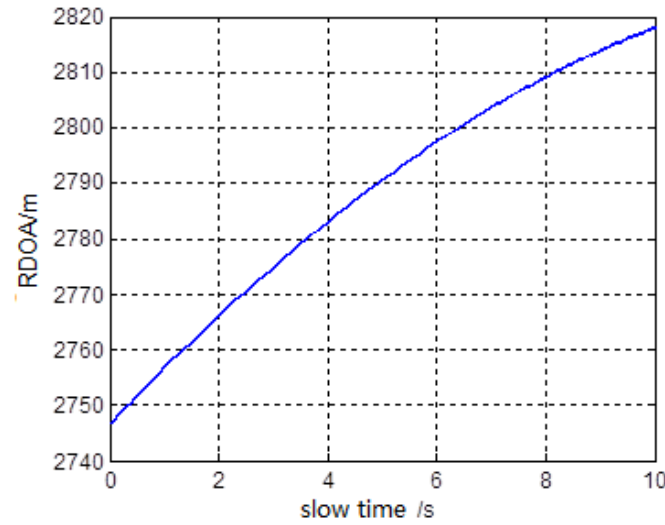

Fig. 2 Changes of RDOA with slow time domain

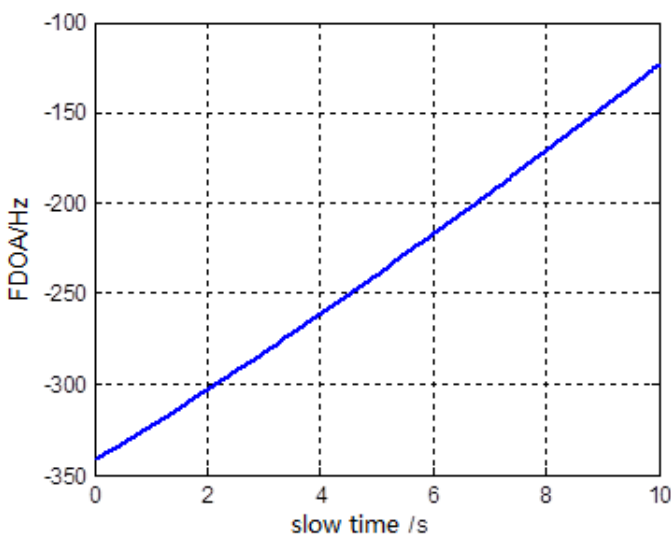

Fig.3 Changes of FDOA with slow time domain

\section{Points Imaging Passive Localization Algorithm}

Assume that the Fourier transformation of the $m$-th pulse signal in fast time domain is $S_{m}\left(f_{r}, t_{m}\right)$, and the spectrum of signals received by two reconnaissance devices in fast time domain are:

$$
\begin{aligned}
& X_{1}\left(f_{r}, t_{m}\right)=S_{m}\left(f_{r}-f_{i}, t_{m}\right) e^{-j 2 \pi f_{r} \tau_{1}\left(t_{m}\right)} e^{-j 2 \pi f_{c} \tau_{1}\left(t_{m}\right)} e^{j 2 \pi f_{i} t_{m}} \\
& X_{2}\left(f_{r}, t_{m}\right)=S_{m}\left(f_{r}-f_{i}, t_{m}\right) e^{-j 2 \pi f_{r} \tau_{2}\left(t_{m}\right)} e^{-j 2 \pi f_{c} \tau_{2}\left(t_{m}\right)} e^{j 2 \pi f_{i} t_{m}}
\end{aligned}
$$

conjugate cross correlation operation is performed to get :

$$
X\left(f_{r}, t_{m}\right)=X_{1}\left(f_{r}, t_{m}\right) \cdot X_{2}^{*}\left(f_{r}, t_{m}\right)=\left|S_{m}\left(f_{r}-f_{i}, t_{m}\right)\right|^{2} \mathrm{e}^{-j \frac{2 \pi f_{r} R\left(t_{m}\right)}{c}} \mathrm{e}^{-j 2 \pi \frac{R\left(t_{m}\right)}{\lambda}}
$$

then the cross ambiguity function[20] of pulses in Fourier transformation domain is: 


$$
\begin{aligned}
& A_{X}\left(t_{r}, t_{m}\right)=\int_{-\infty}^{+\infty} X\left(f_{r}, t_{m}\right) e^{j 2 \pi f_{r} t_{r}} d f_{r} \\
& =\exp \left\{-j 2 \pi \frac{R_{0}+\eta t_{m}+\gamma t_{m}^{2}}{\lambda}\right\} \int_{-\infty}^{+\infty}\left|S_{m}\left(f_{r}-f_{i}, t_{m}\right)\right|^{2} e^{j 2 \pi f_{r}\left(t_{r}-\frac{R_{0}+\eta t_{m}+\gamma t_{m}{ }^{2}}{c}\right)} d f_{r}
\end{aligned}
$$

It can be seen from formula (14) that the maximum of the $A_{X}\left(t_{r}, t_{m}\right)$ is in the fast time domain of $t_{r}=\frac{R_{0}+\eta t_{m}+\gamma t_{m}{ }^{2}}{c}$ and $A_{X}\left(t_{r}, t_{m}\right)$ changes over slow time $t_{m}$. When RDOA walking $\Delta R\left(t_{m}\right)$ exceeds a distance unit, there will be range cell migration to cause defocus and affect the accuracy and resolution of location. However, such RDOA walking is an inherent characteristic in the course of TDOA/FDOA joint estimate position and it's because this RDOA changes over time that makes signals with FDOA property. The traditional way of joint estimation of TDOA and FDOA by cross correlation operation does not take the effects of RDOA walking in TDOA estimation into consideration and there will be fuzzy phenomenon in the corresponding peaks of TDOA and FDOA that causes degradation of accuracy and resolution of estimation. References 21 pointed out that while assuring the RDOA walking of subaperture is less than a distance unit, the RDOA walking can be corrected by center of observing area. Let the RDOA walking corrective term be:

$$
H_{1}\left(f_{r}, t_{m}\right)=\exp \left\{\frac{j 2 \pi}{c} f_{r}\left(\eta_{o} t_{m}+\gamma_{o} t_{m}^{2}\right)\right\}
$$

where $\eta_{o}$ and $\gamma_{o}$ represent linear part and quadratic term part of RDOA walking of $\Delta R\left(t_{m}\right)$ in central observing area $\left(E_{o}\right)$, respectively.

Let the corrected cross correlation function in frequency domain of RDOA walking be:

$$
\begin{aligned}
& X_{C M}\left(f_{r}, t_{m}\right)=X\left(f_{r}, t_{m}\right) \cdot H_{1}\left(f_{r}, t_{m}\right) \\
& =\left|S\left(f_{r}-f_{i}, t_{m}\right)\right|^{2} \mathrm{e}^{-j 2 \pi f_{r} \frac{\left(R_{0}+\left(\eta-\eta_{o}\right) t_{m}+\left(\gamma-\gamma_{o}\right) t_{m}{ }^{2}\right)}{c}} \mathrm{e}^{j 2 \pi \frac{R_{0}}{\lambda}} \mathrm{e}^{-j 2 \pi f_{d} t_{m}} \mathrm{e}^{-j 2 \pi \frac{\gamma t_{m}{ }^{2}}{\lambda}}
\end{aligned}
$$

where $\eta$ denotes the linear part of RDOA walking of target location, $\gamma$ represents the quadratic term part of RDOA walking and $f_{d}$ indicates the TDOA, that $f_{d}=\eta / \lambda$.

There are four exponential terms in formula (16), the first of which is Fourier inverse transformed in fast time domain and then related primarily to peak location as well as can be ignored when $\max \left\{\frac{\left(\eta-\eta_{o}\right) t_{m}+\left(\gamma-\gamma_{o}\right) t_{m}{ }^{2}}{c}\right\}$ is less than a sample time unit. The second exponential term is a constant and the third exponential term is related with FDOA in slow time domain. The forth exponential term is concerned with the quadratic term of RDOA walking in slow time domain, which must be compressed in slow time domain and then the FDOA can be obtained by Fourier inverse transformation.

Let the matched filter compressed by FDOA be:

$$
H_{2}\left(f_{r}, t_{m}\right)=\exp \left\{j 2 \pi \frac{\gamma_{o} t_{m}{ }^{2}}{\lambda}\right\}
$$

The joint estimation of TDOA and FDOA is obtained after compression that the object position is:

$$
A_{c f}\left(t_{r}, f_{m}\right)=\int_{-\infty}^{+\infty} \int_{-\infty}^{+\infty} X_{C C}\left(f_{r}, t_{m}\right) e^{j 2 \pi f_{r} t_{r}} e^{j 2 \pi f_{m} t_{m}} d f_{r} d t_{m}
$$

where

$$
X_{C C}\left(f_{r}, t_{m}\right)=X_{C M}\left(f_{r}, t_{m}\right) \cdot H_{2}\left(f_{r}, t_{m}\right) .
$$

From the formula (18), we can get the TDOA $\left(\hat{t}_{r}\right)$ and the FDOA $\left(\hat{f}_{m}\right)$ of emitter to two 
reconnaissance devices and by geometric relation, the mapped target location is:

$$
\begin{aligned}
& \hat{x}_{T}=\frac{2 \hat{R}_{10} c \hat{t}_{r}-\left(c \hat{t}_{r}\right)^{2}}{4 L} \\
& \hat{y}_{T}=\frac{\hat{f}_{m} \lambda \hat{R}_{10}\left(\hat{R}_{10}-c \hat{t}_{r}\right)}{\left(v_{1}-v_{2}\right) \hat{R}_{10}-v_{1} c \hat{t}_{r}}
\end{aligned}
$$

and the specific solving process of $\hat{R}_{10}$ is given in appendix.

\section{Simulation Analysis}

In order to validate the validity of the proposed algorithm, the simulation experiment were carried out under single target's and multiple target's circumstances.

Assuming the center location of observing area is in $[6 \mathrm{Km}, 2 \mathrm{Km}, 0]^{\mathrm{T}}$, reconnaissance aircraft $P_{1}$ and reconnaissance aircraft $P_{2}$, which both fly along the $\mathrm{Y}$ axis at the speed of $200 \mathrm{~m} / \mathrm{s}$, are located in $[-2.5 \mathrm{Km}, 0,10 \mathrm{Km}]^{\mathrm{T}}$ and in $[2.5 \mathrm{Km}, 0,10 \mathrm{Km}]^{\mathrm{T}}$ at the time of $t=0$ respectively. The sampling rate of reconnaissance receiver is $f_{s}=100 \mathrm{MHz}$, the samples is $N=1024$ in a time slice and the sample signal's ratio of signal-to-noise is $S N R=10 \mathrm{~dB}$.

Figure 4 gives the points imaging passive location under single target condition, and the detailed parameter settings are as follows: the position coordinate of emitter is $[7.0,3.0,0]^{\mathrm{T}} \mathrm{km}$ and transmits linear frequency modulation signal, the carrier frequency is $f_{c}=10 \mathrm{GHz}$, the bandwidth is $B=80 \mathrm{MHz}$ and the pulse width is $T_{r}=4 \mu \mathrm{s}$.

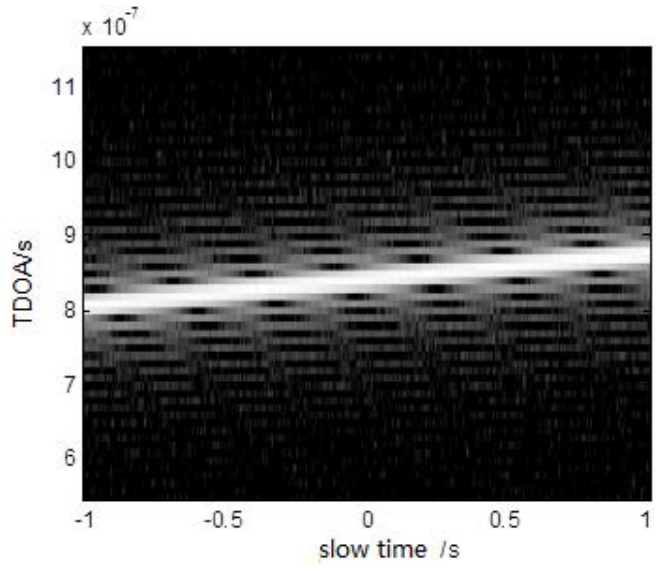

(a) The RDOA walking parameters (before correction)

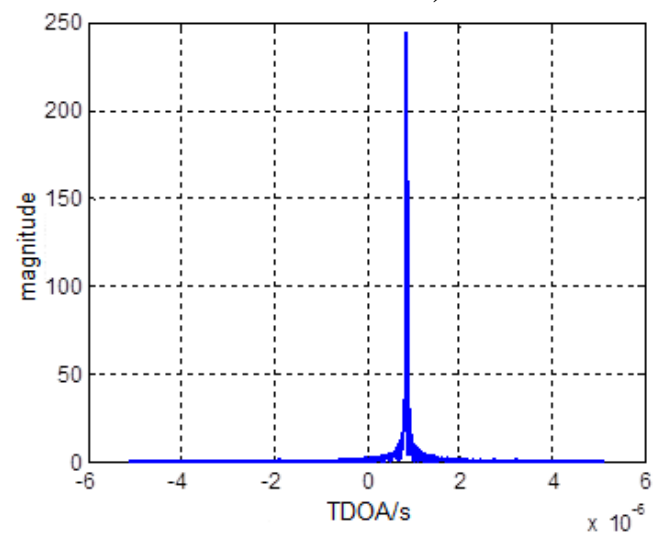

(c) The TDOA profile after corrected compression

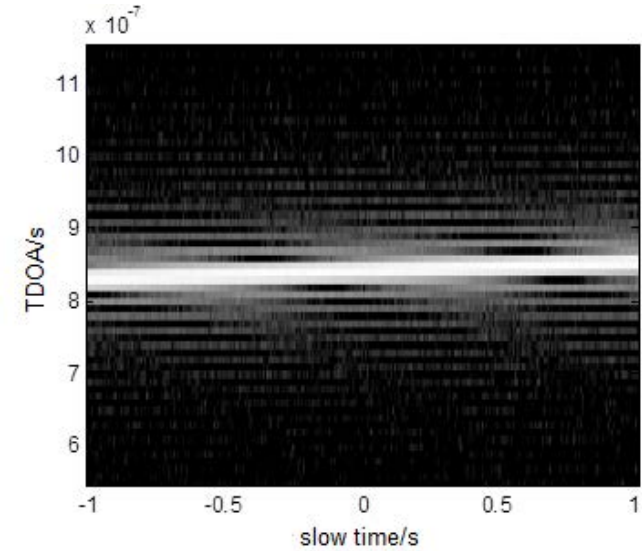

(b) The RDOA walking parameters (after correction)

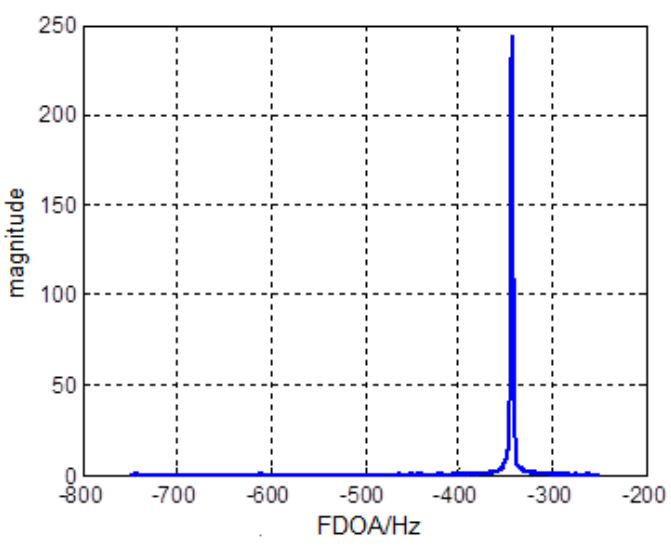

(d) The FDOA profile after corrected 


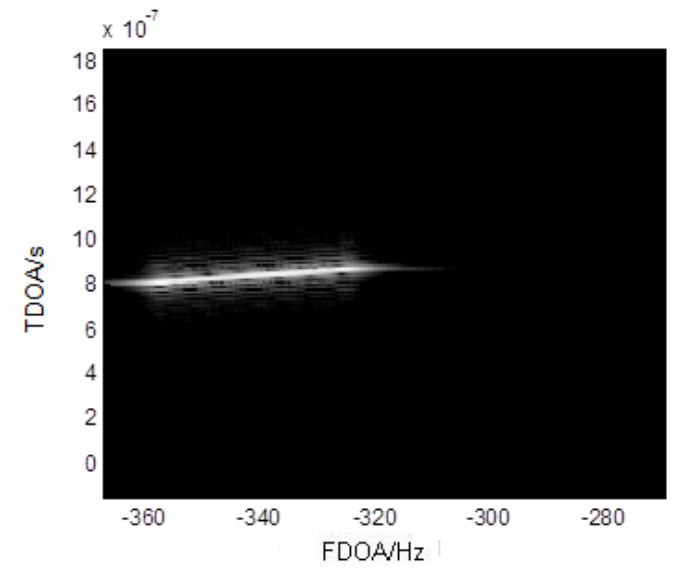

(e) The localization results of uncorrected compression

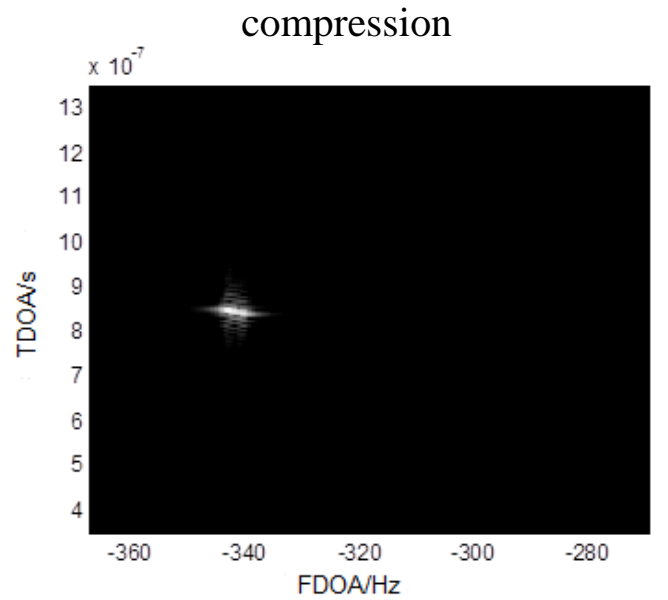

( $f$ ) The localization results of corrected compression

Fig. 4 The results of single objective points imaging passive localization

The changes of the TDOA at FDOA direction in slow time domain in the case of RDOA walking parameters (before correction) is presented in Figure 4(a). As can be seen from Figure 4(a), TDOA changes with slow time and spans multiple range cells. Figure 4(b) gives the changes of the TDOA in slow time domain in the case of RDOA walking parameters (after correction), which TDOA remains unchanged basically that can be considered in the same range cell. The TDOA profile after corrected compression and the FDOA profile after corrected compression are shown in Figure $4(c)$ and $4(d)$ respectively and good focusing effect are obtained both in TDOA and in FDOA. The positioning result before corrected compression is presented in Figure 4(e), whereas Figure 4(f) gives the localization result after corrected compression. A fuzzy band is presented in Figure 4(e), which spans multiple units at TDOA and FDOA and vague problems exist in estimating TDOA and FDOA. Compared with Figure 4(e), Figure 4(f) has obviously improved, which basically stays in the same unit that can achieve better estimation accuracy and resolution. The TDOA and the FDOA of bright spot in Figure $4(f)$ is $9.1633 \mu$ s, and $-343.2617 \mathrm{~Hz}$, respectively. The location of target point mapped by the bright spot in Figure $4(f)$ is $[7.009,3.009,0]^{\mathrm{T}} \mathrm{km}$. The above simulation results show that the effect of the proposed points imaging passive localization algorithm.

Both Figure 5 and 6 are the results of points imaging passive localization in the case of three emitters. All these three emitters lunch linear frequency modulated signal with the same carrier frequency ( $f_{c}=10 \mathrm{GHz}$ ), bandwidth are $B_{1}=60 \mathrm{MHz}, B_{2}=70 \mathrm{MHz}$ and $B_{3}=80 \mathrm{MHz}$ respectively , in addition, pulse width are $T_{r 1}=8 \mu \mathrm{s}, T_{r 2}=9 \mu \mathrm{s}$ and $T_{r 3}=10 \mu \mathrm{s}$ respectively. The coordinates of three emitters in Figure 5 are $[7.0,3.0,0]^{\mathrm{T}} \mathrm{km},[7.0,2.9,0]^{\mathrm{T}} \mathrm{km}$ and $[7.0,3.1,0]^{\mathrm{T}} \mathrm{km}$ respectively. The coordinates of three emitters in Figure 6 are $[7.0,3.0,0]^{\mathrm{T}} \mathrm{km}, \quad[6.9,3.0,0]^{\mathrm{T}} \mathrm{km}$ and $[7.1,3.0,0]^{\mathrm{T}} \mathrm{km}$, respectively. 


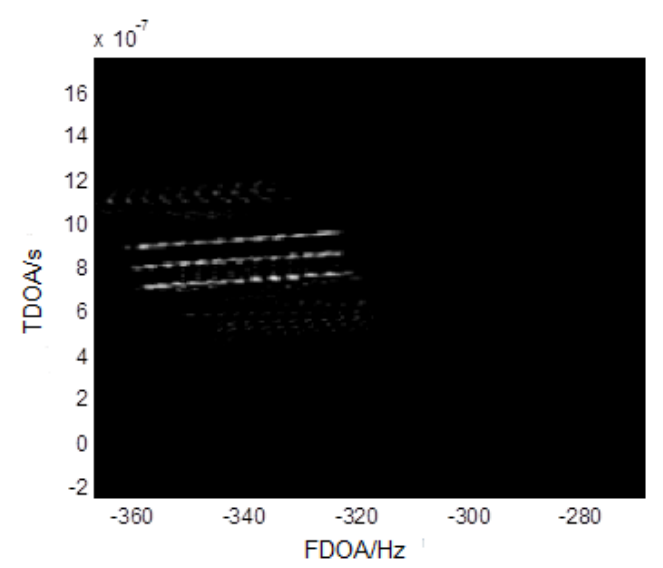

(a) The localization result before corrected compression

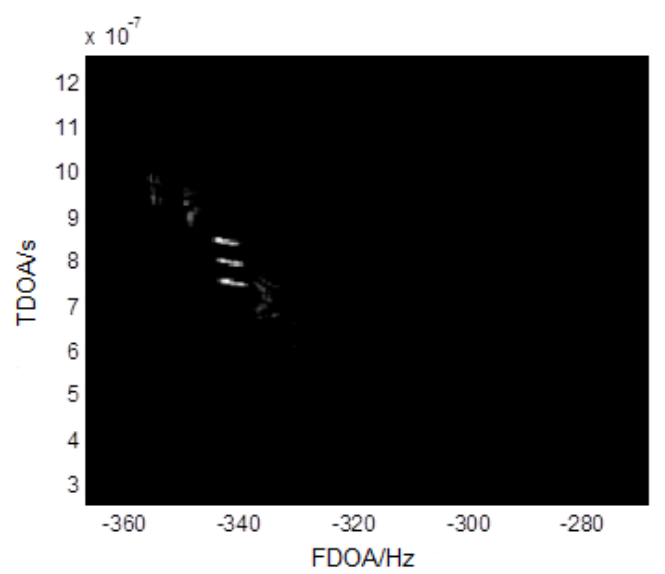

(b) The localization result after corrected compression

Fig.5 The imaging passive localization results of different three target points in $\mathrm{X}$ axis

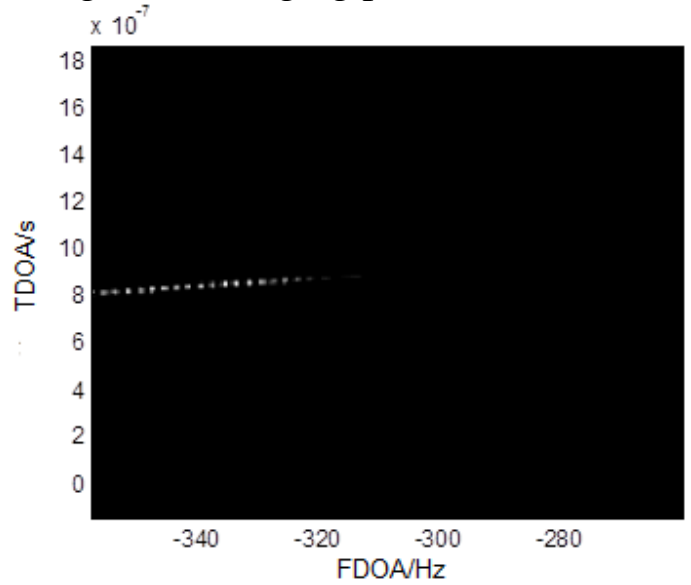

(a) The localization result before corrected compression

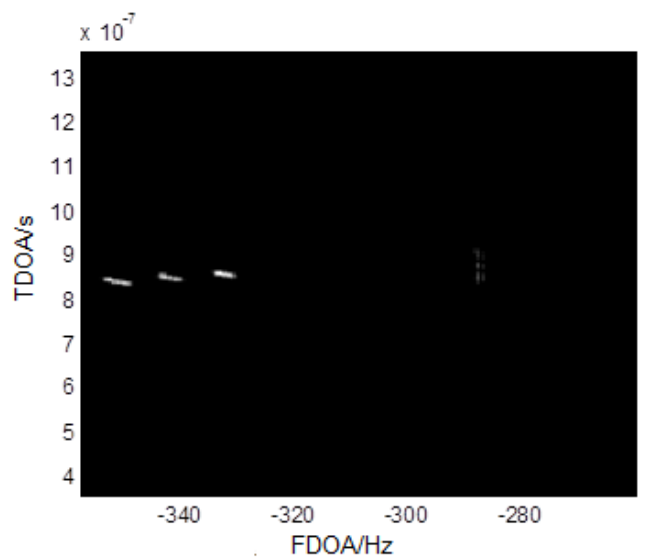

(b) The localization result after corrected compression

Fig.6 The imaging passive localization results of different three target points in Y axis.

Figure 5(a) and 6(a) are the localization results before corrected compression. Three targets and three banded bright lines could still be distinguished in Figure 5(a), however, there is only one bright line in Figure 6(a) that couldn't distinguish the number and the location of targets. Both Figure $5(b)$ and $6(b)$ are the localization by corrected compression and can well recognize three targets. These three targets location corresponding to bright spots in Figure $5(b)$ are $[6.913,3.011,0]^{\mathrm{T}} \mathrm{km},[7.107,3.008,0]^{\mathrm{T}} \mathrm{km}$ and $[6.887,2.987,0]^{\mathrm{T}} \mathrm{km}$, respectively. These three targets location corresponding to bright spots in Figure $6(b)$ are $[7.001,2.907,0]^{\mathrm{T}} \mathrm{km}$, $[7.007,3.113,0]^{\mathrm{T}} \mathrm{km}$ and $[6.887,2.987,0]^{\mathrm{T}} \mathrm{km}$, respectively. As shown in Figure 5 and 6 , the points imaging passive localization algorithm described in this paper applies to multiple emitters localization as well and has high location accuracy and resolution.

\section{Discussion}

Aiming at the problem of passive reconnaissance and localization of wideband signal source, a points imaging passive localization algorithm for bistatic stations in the parallel track and squint mode based on SAR imaging theory and direct position determination is proposed in this paper. On the basis of analyzing RDOA walking in the parallel track and squint mode, the RDOA walking is corrected and the FDOA spatial variation is compressed, then TDOA and FDOA are mapped to the position of targets by joint estimation. This method does not require two-dimensional searching for 
target location and has low computation. For multi-target localization, there is no need for signal sorting and parameter pairing, which is applicable for observing indistinguishable multi-target location problems. The simulation experiment is provided to illustrate the effectiveness of the proposed algorithm, which also has high-precision and super resolution properties.

\section{Appendix Solution of $R_{10}$}

To simplify the computation, let:

$$
\begin{gathered}
K=f_{d} \lambda \\
\Delta v=v_{1}-v_{2}
\end{gathered}
$$

Based on equations of TDOA and FDOA, we can get:

$$
\begin{aligned}
& x_{T}+L=k_{1}+k_{2} R_{10} \\
& y_{T}=\frac{K R_{10}\left(R_{10}-R_{0}\right)}{\Delta v \cdot R_{10}-v_{1} R_{0}}
\end{aligned}
$$

where,

$$
\begin{aligned}
& k_{1}=L-\frac{R_{0}^{2}}{4 L} \\
& k_{2}=\frac{R_{0}}{2 L}
\end{aligned} .
$$

Plug the equations (22) and (23) into $R_{10}$, the obtained quartic equation is:

$$
m_{1} R_{10}{ }^{4}+m_{2} R_{10}{ }^{3}+m_{3} R_{10}{ }^{2}+m_{4} R_{10}+m_{5}=0
$$

where,

$$
\begin{aligned}
& m_{1}=K^{2}-\left(1-k_{2}^{2}\right) \Delta v^{2} \\
& m_{2}=-2 K^{2} R_{0}+2\left(1-k_{2}^{2}\right) R_{0} \cdot \Delta v \cdot v_{1}+2 k_{1} k_{2} \Delta v^{2} \\
& m_{3}=K^{2} R_{0}^{2}-\left(1-k_{2}^{2}\right) v_{1}^{2} R_{0}^{2}-4 k_{1} k_{2} R_{0} \cdot \Delta v \cdot v_{1}+\left(k_{1}^{2}+H_{1}^{2}\right) \Delta v^{2}, \\
& m_{4}=2 k_{1} k_{2} v_{1}^{2} R_{0}^{2}-2\left(k_{1}^{2}+H_{1}^{2}\right) R_{0} \cdot \Delta v \cdot v_{1} \\
& m_{5}=\left(k_{1}^{2}+H_{1}^{2}\right) v_{1}^{2} R_{0}^{2}
\end{aligned}
$$

$R_{10}$ can be obtained by solving the quartic equation.

\section{Acknowledgement}

In this paper, the research was sponsored by the National Natural Science Foundation of China under grant 61171170 and the Natural Scientific Fundamental Research Project of Shanxi Province of China (No. 2013JQ8020).

\section{References}

[1] Zhou C, Huang G M, Shan H C, et al. Bias compensation algorithm based on maximum likelihood estimation for passive localization using TDOA and FDOA measurement [J]. Acta Aeronautica et Astronautica Sinica, 2015, 36(3): 979-986 (in Chinese).

[2] Guo Huizhu, Da Zhengfeng. Bi-iterative method for moving source localisation using TDOA and FDOA measurements [J]. Electronics Letters, 2015, 51(1): 8-10.

[3] Oren Jean, Anthony J. Passive Localization and synchronization using arbitrary signals [J]. IEEE Transactions on Signal Processing, 2014, 62(8): 2143-2150.

[4] Weiss A J. Direct position determination of narrowband radio frequency transmitters[J]. IEEE Signal Processing Letters, 2004,11(5):513-516.

[5] Amar A, Weiss A J. Advances in direct position determination[C]. Sensor Array and Multichannel Signal Processing Workshop Proceedings, 2004:584-588. 
[6] Amar A, Weiss A J. Analysis of direct position determination approach in the presence of model errors[C]. IEEE/SP 13th Workshop on Statistical Signal Processing, Novosibirsk, 2005:521-524.

[7] Amar A, Weiss A J. Direct position determination of multiple radio signals[C] IEEE International Conference on Acoustics, Speech, and Signal Processing, 2004,2:81-84.

[8] Weiss A J, Amar A. Direct geolocation of stationary wideband radio signal based on time delays and Doppler shifts[C]. IEEE/SP 15th Workshop on Statistical Signal Processing, Cardiff, 2009:101-104.

[9] Weiss A J, Amar A. Direct geolocation of wideband emitters based on delay and Doppler[J]. IEEE Transactions on Signal Processing, 2011,59(6):2513-2521.

[10]Pourhomayoun M, Flowler M L. Sensor network distributed computation for direct position determination[C]. IEEE 7th Sensor Array and Multichannel Signal Processing Workshop (SAM), Hoboken, NJ, 2012:125-128.

[11] Mohammad Pourhomayoun, Mark L. Fowler. Distributed computation for direct position determination emitter location[J]. IEEE Transaction on Aerospace and Electronic System, 2014, 50(4): 2878-2889.

[12]Zhang M, Guo F C, Zhou Y Y. A Single Moving observer direct position determination method using a long baseline interferometer [J]. Acta Aeronautica et Astronautica Sinica, 2013, 34(2): 378-386 (in Chinese).

[13]V. Cevher, M. F Duarte, R.G Baraniuk. Distributed target localization via spatial sparsity[C]. $16^{\text {th }}$ European Signal Processing Conference, Switzerland, 2008,25-29.

[14]W. Ke, T.T. Wang, L.N. Wu. Multi-target direct localization via approximate $l_{0}$ norm minimization [J]. Electronics letters. 2012,48(23):1498-1500.

[15]Pourhomayoun M, Fowler M L, Wu N E. Spatial sparsity based emitter localization[C]. The 46th Annual Conference on Information Sciences and Systems (CISS), Princeton, NJ, 2012:1-4.

[16]I. G. Cumming, F. H. Wong. Digital processing of synthetic aperture radar data: algorithms and implementation [M]. Norwood, MA: Artech House, 2005.

[17]W. M. Brown and L. J. Porcello. An introduction to synthetic aperture radar[J]. IEEE Spectrum, 1969, 6(9) ; 52-62.

[18]C. A. Wiley. Synthetic aperture radar[J]. IEEE Trans. On AES, 1985, 21(3): 440-443.

[19]TANG Z Y, ZHANG S R. Reference frame of bistatic synthetic aperture radar [M]. Beijing: Science and Technology Press, 2003 (in Chinese).

[20]ZHANG X D. Modern signal processing[M]. Beijing: Tsinhua University Press, 2002(in Chinese).

[21] Zhou Peng. Study on imaging algorithm for missile-borne SAR in multiple modes [D]. Xi'an: XiDian University, 2011 (in Chinese). 